\title{
Variations saisonnières de l'œstrus et de l'ovulation chez la chèvre locale Maure en Tunisie
}

\author{
N. Lassoued ${ }^{1 *}$ M. Rekik ${ }^{2}$
}

Mots-clés

Caprin - Chèvre - Cycle œstral Ovulation - Saison d'accouplement Reproduction - Tunisie.

\begin{abstract}
Résumé
Un total de 22 chèvres Maures tunisiennes ont permis de caractériser les

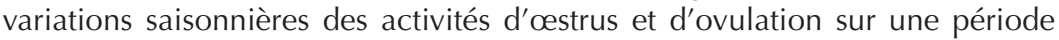
de 14 mois. Durant la période expérimentale, les chèvres ont été maintenues non gravides. Elles ont été conduites sur des parcours naturels et ont reçu un apport constant en concentré de $200 \mathrm{~g}$ par chèvre et par jour. En outre, elles ont été exposées à une photopériode naturelle et maintenues en présence continue des boucs. Des boucs munis de tabliers ont été utilisés pour la détection biquotidienne du comportement d'œstrus, alors que l'examen de l'activité ovarienne a été réalisé par des endoscopies mensuelles. Au cours de la saison sexuelle, qui s'est étendue de septembre à mars, 80 p. 100 des chèvres ont montré un comportement d'œstrus au moins une fois et 53 p. 100 de la totalité des cycles œestriens ont été accompagnés d'ovulation. Cette saison a précédé une période d'inactivité sexuelle de mars à août. L'incidence des cycles œstriens anormaux de courte durée a été plus élevée vers la fin de la saison sexuelle. Pendant le mois de septembre, 86 p. 100 des chèvres ont montré un comportement $\mathrm{d}^{\prime} œ$ œtrus. La même distribution saisonnière a été aussi observée pour l'activité cyclique ovarienne. La proportion de femelles ovulant pendant la saison sexuelle a augmenté progressivement à partir du mois de septembre pour atteindre un maximum de 100 p. 100 en décembre et a commencé ensuite à baisser pour atteindre un minimum de 14 p. 100 au mois de mars. La valeur moyenne du taux d'ovulation a été de $1,51 \pm 0,29$, atteignant un maximum de 1,94 $\pm 0,74$ au début de la saison sexuelle en septembre et un minimum de 1,25 $\pm 0,50$ à la fin de cette saison, au mois de mars. Le taux d'ovulation a été nul entre les mois d'avril et d'août inclus. L'écart entre le début et la fin de la saison sexuelle a été significativement différent $(P<0,05)$. En conclusion, les chèvres locales en Tunisie, sous un milieu semi-aride, ont un rythme reproductif saisonnier.
\end{abstract}

\section{INTRODUCTION}

L'existence des variations de l'activité sexuelle au cours d'une année a été rapportée chez plusieurs races ovines et caprines dans le monde. C'est une donnée fondamentale sur la physiologie de la reproduction des chèvres dont la connaissance est très importante pour conduire la reproduction, que cela soit par saillie naturelle ou par insémination artificielle. Ces variations plus ou moins importantes dépendent surtout des races et aussi des facteurs de l'environnement,

\footnotetext{
1. Institut national de recherche agronomique de Tunisie, rue Hédi Karray, 2080 Ariana, Tunisie

2. Ecole nationale de médecine vétérinaire, 2020 Sidi Thabet, Tunisie

* Auteur pour la correspondance

Tél. : +21671230 239; fax : +21671 752897

E-mail : lassoued.narjess@iresa.agrinet.tn
}

principalement la lumière, la température, l'alimentation, avec des interactions entre individus telles que la présence ou l'absence des mâles. Dans les pays tempérés, la photopériode et l'amplitude de ses changements constituent le facteur principal déterminant l'existence ou l'absence d'activité sexuelle chez les ovins et les caprins (12). Sous des conditions tropicales et autour de l'équateur où ces changements sont faibles, il est connu que les races locales d'ovins et de caprins peuvent se reproduire toute l'année ou bien présentent une faible saisonnalité (8) avec des baisses de fertilité (9). Toutefois, il existe des variations importantes entre les races vivant sous la même photopériode d'une part (3) et des variations intra-race d'autre part. En effet, les différences entre individus peuvent être importantes (11). En Tunisie, certains travaux se sont intéressés à l'étude des variations saisonnières de l'activité sexuelle des ovins à viande de races Barbarine (13), Noire de Thibar et Queue fine de l'Ouest (15). Ces races locales ont une saison sexuelle qui s'étend de la fin du mois de 
juillet jusqu'au mois de février. En dehors de cette période, un certain pourcentage (50 p. 100 en moyenne) des femelles maintiennent une activité sexuelle. Les caractéristiques de reproduction des chèvres locales ont été très peu étudiées. Pourtant, le choix des stratégies et des méthodes de reproduction qui peuvent contribuer à l'amélioration de la productivité des troupeaux caprins nécessite la connaissance approfondie des variations saisonnières de l'activité sexuelle. Ainsi, le présent travail a eu pour objectif de déterminer les caractéristiques des variations de l'activité sexuelle chez la chèvre Maure de Tunisie.

\section{MATERIEL ET METHODES}

Cette étude a eu lieu à la station expérimentale de Ouesslatia, appartenant à l'Institut national de la recherche agronomique de Tunisie. Cette station est située à $200 \mathrm{~km}$ au sud-ouest de Tunis $\left(35^{\circ} 51^{\prime} \mathrm{N}\right.$ et $\left.9^{\circ} 35^{\prime} \mathrm{E}\right)$ sous un climat de type semi-aride. Les données climatiques (température, humidité, pluviométrie et durée d'ensoleillement), fournies par l'Institut national de la météorologie de Tunisie, représentent une moyenne mensuelle de 30 années successives (1973-2003 ; tableau I) et la moyenne de la période d'étude (tableau II, figure 1). La pluviométrie annuelle moyenne est de $400 \mathrm{~mm}$ et caractérisée par sa répartition saisonnière extrêmement irrégulière.

Vingt-cinq chèvres âgées de 3 à 6 ans et ayant un poids vif moyen de $38,5 \pm 3,1 \mathrm{~kg}$ ont été utilisées dans cette étude. Les chèvres ont été maintenues hors reproduction pendant la période de l'étude qui a

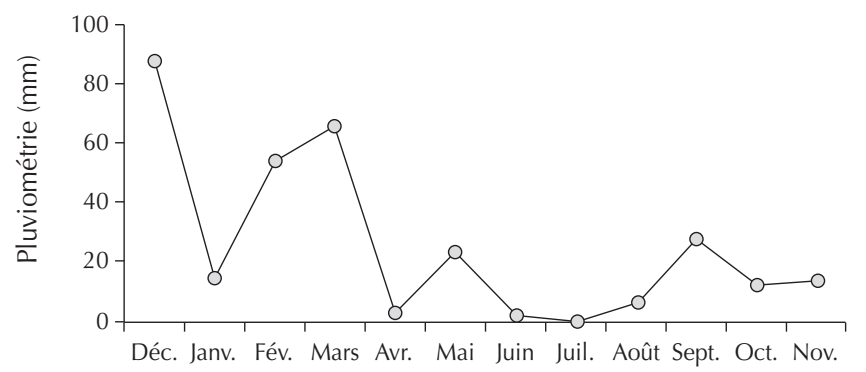

Figure 1 : pluviométrie moyenne au cours de l'année d'étude. duré 14 mois. Elles ont été conduites sur des parcours naturels essentiellement à base d'armoise blanche, armoise champêtre, lentisque et romarin. Elles ont pâturé quotidiennement pendant $5 \mathrm{~h}$ et ont reçu en plus $200 \mathrm{~g}$ d'un concentré commercial par tête et par jour.

Les animaux ont été conduits en permanence sous une photopériode naturelle en présence de sept boucs adultes de la même race. Sur parcours, les boucs ont été munis de tabliers et en bergerie la séparation entre mâles et femelles a été faite de manière à maintenir la vue et l'odorat.

L'activité ovarienne a été contrôlée par des endoscopies mensuelles, tous les 15 du mois, selon la technique décrite par Thimonier et Mauléon (25) et l'âge des corps jaunes a été défini par leur taille et leur couleur (18). Le taux d'ovulation est défini comme étant le nombre moyen d'ovulations par cycle déduit du nombre de corps jaunes observés. Le contrôle des chaleurs a été effectué quotidiennement deux fois par jour à $6 \mathrm{~h}$ et $18 \mathrm{~h}$ pendant une heure avec des boucs munis de tabliers. Le critère d'œstrus est l'immobilisation de la femelle sous le chevauchement par le mâle (17).

La comparaison des proportions de femelles dans différentes catégories a été réalisée par un test du chi-2. Lorsque le nombre d'observations pour une catégorie était inférieur à cinq, le test exact de Fisher était utilisé. La comparaison des moyennes du taux d'ovulation pour les différents mois de la saison sexuelle a été réalisée par un test $t$ de Student.

\section{RESULTATS}

Dès le début de l'étude, deux chèvres ont été saillies par erreur et ont été écartées après la deuxième endoscopie. Une autre chèvre a été éliminée à la quatrième endoscopie après avoir développé des adhérences au niveau de l'ovaire gauche qui ont rendu les observations impossibles. Les résultats présentés ont donc porté sur 22 chèvres.

\section{Variations saisonnières du comportement d'œestrus}

A peu près le quart des chèvres (26 p. 100) ont commencé la saison sexuelle au mois de septembre par un comportement d'œstrus sans

\section{Tableau I}

Moyennes mensuelles de la température, de l'humidité et de la pluviométrie sur 30 ans (Institut national de la météorologie)

\begin{tabular}{|c|c|c|c|c|c|c|c|c|c|c|c|c|c|}
\hline & Janv. & Fév. & Mars & Avr. & Mai & Juin & Juil. & Août & Sept. & Oct. & Nov. & Déc. & Total \\
\hline $\mathrm{T}$ & 9,2 & 9,9 & 11,9 & 14,8 & 18,8 & 23,1 & 26,3 & 26,5 & 22,9 & 18,4 & 13,7 & 10,3 & - \\
\hline $\mathrm{H}$ & 68,5 & 66,8 & 63,5 & 60,6 & 55,5 & 52,6 & 52,0 & 58,0 & 63,2 & 65,3 & 64,8 & 68,3 & - \\
\hline PL & 43,3 & 34,2 & 41,1 & 39,2 & 34,7 & 16,0 & 7,6 & 20,3 & 58,2 & 47,6 & 29,0 & 28,5 & 399,7 \\
\hline
\end{tabular}

$\mathrm{T}$ : température $\left({ }^{\circ} \mathrm{C}\right) . \mathrm{H}$ : humidité $(\%)$. PL : pluviométrie $(\mathrm{mm})$

\section{Tableau II}

Durée d'ensoleillement, température et pluviométrie pendant la période de l'étude

\begin{tabular}{|c|c|c|c|c|c|c|c|c|c|c|c|c|c|c|}
\hline & Oct. & Nov. & Déc. & Janv. & Fév. & Mars & Avr. & Mai & Juin & Juil. & Août & Sept. & Oct. & Nov. \\
\hline$E$ & 7,3 & 6,3 & 5,1 & 6,1 & 4,5 & 6,7 & 9,4 & 9,5 & 9,9 & 10,9 & 10,6 & 8,1 & 6,9 & 6,5 \\
\hline $\mathrm{T}$ & 19,9 & 12,2 & 9,9 & 8,1 & 8,0 & 10,3 & 14,7 & 18,9 & 23,9 & 26,1 & 27,2 & 23,6 & 19,8 & 13,6 \\
\hline $\mathrm{P}$ & 21,9 & 111,5 & 87,5 & 14,8 & 53,8 & 65,6 & 2,2 & 23,3 & 2,0 & 0,0 & 6,2 & 27,8 & 12,2 & 13,6 \\
\hline
\end{tabular}

E : durée d'ensoleillement $(\mathrm{h} / \mathrm{j})$. T : température $\left({ }^{\circ} \mathrm{C}\right) . \mathrm{P}$ : pluviométrie $(\mathrm{mm})$ 
ovulation préalable, ni accompagnant l'œstrus. En moyenne, du mois de septembre au mois de mars, 80 p. 100 des femelles caprines ont extériorisé un comportement de chaleur. L'arrêt de l'activité œstrienne a été progressif entre février et la fin du mois de mars (figure 2).

Au cours du mois de septembre, 86 p. 100 des femelles ont montré au moins un comportement d'œstrus (figure 2). Parmi ces femelles, 47 p. 100 ont extériorisé plus d'un comportement presque dans tous les cas (8/9) séparé par un cycle de courte durée. Sur la totalité des œstrus dénombrés dans cette étude, seulement la moitié (53 p. 100) ont été accompagnés d'une ovulation. Les cycles œstriens de courte durée ont été observés au début (septembre) et d'une manière plus accentuée à la fin de la saison sexuelle (de janvier à mars) avec 68 p. 100 des chèvres montrant des cycles courts intercalés dans une série de comportements d'œstrus.

\section{Variations saisonnières de l'activité ovulatoire}

Les femelles ont commencé à ovuler à partir du mois de septembre et les ovulations se sont maintenues jusqu'au mois de mars. Au cours de cette période d'automne hiver, qui coïncide avec la saison des pluies (figure 1), le maximum de chèvres cycliques s'est situé en décembre (100 p. 100) et le minimum en mars (14 p. 100) avec une moyenne générale de l'ordre de 76 p. 100 tout au long de la saison sexuelle (figure 2).

Si nous considérons la moyenne des ovulations silencieuses (non accompagnées d'œstrus) ou des œstrus non accompagnés d'ovulation ou des ovulations accompagnées de comportement de chaleurs, nous déduisons qu'il existe une très large variabilité entre les chèvres. Toutefois, le pourcentage d'ovulations accompagnées d'œstrus a présenté des variations durant l'année (figure 3). Il a atteint un maximum de 69 p. 100 pendant le mois d'octobre et a diminué à partir du mois de janvier.

\section{Caractéristiques des cycles sexuels}

Sur les 22 chèvres, 19 ont manifesté des œstrus détectés pendant l'étude. Plusieurs types de cycles sexuels ont été observés et se caractérisaient généralement par une dissociation des cycles œstriens et des cycles ovulatoires. En effet, il y a eu des cycles sexuels délimités par deux œstrus, des cycles sexuels délimités par deux ovulations et des cycles normaux délimités par des ovulations accompagnées d'œstrus. Dans ce dernier type, et durant toute la période d'activité sexuelle, il a été dénombré un total de 26 cycles, soit en moyenne 1,2 cycle normal par chèvre et par saison sexuelle. La durée moyenne de ce type de cycles a été de 21,1 \pm 1,5 jours.

\section{Variations saisonnières du taux d'ovulation}

La moyenne annuelle du taux d'ovulation a été de 1,51 \pm 0,29. Au cours de la saison d'activité ovarienne, le taux d'ovulation a présenté un maximum au mois de septembre en début de la saison sexuelle (tableau III). Par la suite, le taux d'ovulation a diminué progressivement, mais avec une différence significative $(\mathrm{P}<0,05)$ entre le début (septembre-octobre) et la fin (janvier-février-mars) de la saison sexuelle (tableau III).

\section{DISCUSSION}

Les résultats de cette étude ont montré que l'activité sexuelle des femelles caprines de la race locale Maure de Tunisie était très saisonnée et limitée entre mi-septembre et fin février. En dehors de cette période d'automne hiver, il n'y a eu aucune activité ostrienne ou ovulatoire. Cette variation de l'activité ovarienne a été similaire à celle de la chèvre Bédouine algérienne (4) également des régions subtropicales, et à celle de la chèvre Alpine (7) des régions tempérées. Toutefois, elle a été différente de celle de la chèvre D'Man au Maroc qui présente une activité continue avec une baisse de fertilité au printemps (9). Cette baisse est due à une diminution de l'activité ovarienne et de la manifestation du comportement de chaleur. En Guadeloupe, la chèvre Créole montre également une activité œstrienne et/ou ovulatoire pendant toute l'année (6). Le tableau IV montre quelques exemples de saisonnalité de l'activité sexuelle de différentes races caprines dans les zones tropicales, subtropicales et tempérées.

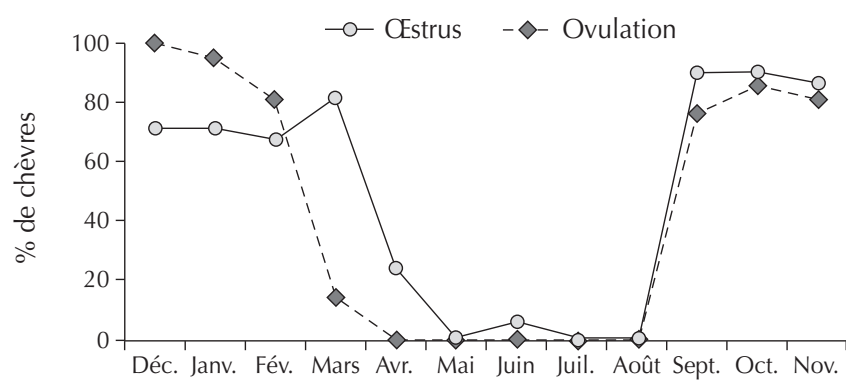

Figure 2 : évolution du pourcentage de chèvres ovulant ou montrant un comportement d'œstrus au moins une fois par mois.

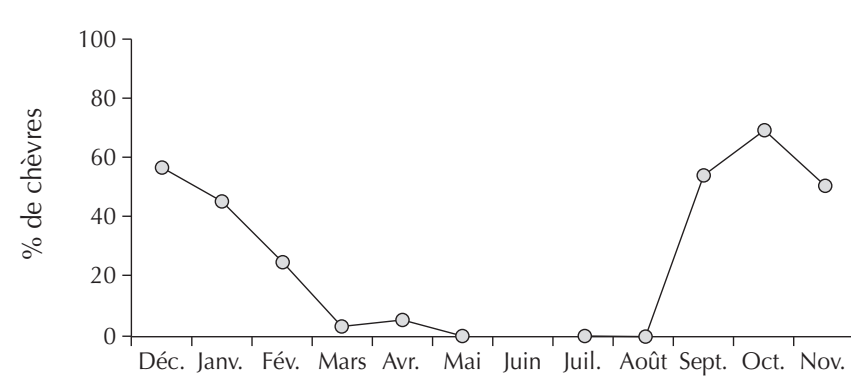

Figure 3 : variations saisonnières du pourcentage de chèvres montrant une ovulation accompagnée d'œstrus.

\section{Tableau III}

Evolution du taux d'ovulation moyen des chèvres locales Maures en Tunisie pendant les mois d'activité ovulatoire $(n=22)$

\begin{tabular}{lccccccc} 
& Sept. & Oct. & Nov. & Déc. & Janv. & Fév. & Mars \\
\hline Nb. de chèvres ayant ovulé & 17 & 20 & 17 & 22 & 20 & 15 & 4 \\
Taux d'ovulation & 1,94 & 1,60 & 1,41 & 1,43 & $1,27 \pm$ & 1,21 & 1,25 \\
& $\pm 0,74^{\mathrm{a}}$ & $\pm 0,49 \mathrm{ab}$ & $\pm 0,51^{\mathrm{bc}}$ & $\pm 0,59 \mathrm{bc}$ & $0,45^{\mathrm{c}}$ & $\pm 0,59^{\mathrm{c}}$ & $\pm 0,50^{\mathrm{bc}}$
\end{tabular}

a,b,c Les valeurs moyennes du taux d'ovulation portant une même lettre ne sont pas significativement différentes $(P>0,05)$ 


\section{Tableau IV}

Quelques exemples de saisonnalité de l'activité sexuelle de différentes races caprines dans les zones tropicales, subtropicales et tempérées

$\begin{array}{lllll}\text { Cyclicité } & \text { Pays } & \text { Race } & \text { Saison sexuelle } & \text { Référence } \\ \text { Non continue } & \text { France } & \text { Alpine } & \text { Septembre-février } & \text { Chemineau et coll., 1992, Small Rumin. Res. } \\ \text { Non continue } & \text { Tunisie } & \text { Locale Maure } & \text { Septembre-mars } & \text { Présent travail } \\ \text { Non continue } & \text { Algérie } & \text { Bédouine } & \text { Fin été à fin hiver } & \text { Charallah et coll., 2000, Conf. int. Caprins } \\ \text { Non continue } & \text { Argentine } & \text { Créole } & \text { Janvier-novembre } & \text { Rivera et coll., 2003, Small Rumin. Res. } \\ \text { Continue } & \text { Guadeloupe } & \text { Créole } & \text { Toute l'année } & \text { Chemineau, 1986, Reprod. Nutr. Dev. } \\ \text { Continue } & \text { Maroc } & \text { D'Man } & \text { Toute l'année } & \text { Derquaoui et El Khaledi, 1994, Cipea }\end{array}$

Contrairement donc à la majorité des races ovines de l'Afrique du Nord, qui maintiennent une activité sexuelle toute l'année, la chèvre locale Maure en Tunisie est un animal dont la reproduction est saisonnière. En effet, en Tunisie, 40 à 50 p. 100 des brebis de races Barbarine (13), Noire de Thibar et Queue fine de l'Ouest (15) continuent à ovuler et à avoir un comportement d'œstrus au cours du printemps et au début de l'été. Les races ovines Tadmit en Algérie (1) ou D’Man au Maroc (14) présentent une activité sexuelle continue pendant l'année. Ce caractère saisonnier très accentué des rythmes reproductifs des chèvres locales en Tunisie est d'ailleurs confirmé par nos observations antérieures par endoscopies de leur activité ovarienne aux mois d'avril et mai et par la réponse à l'effet bouc à la même période (travaux non publiés dans la même station expérimentale). En effet, pendant dix années consécutives, aux mois d'avril et de mai, aucune chèvre du troupeau n'a été spontanément cyclique avant l'introduction des boucs, indiquant une forte intensité d'anœstrus (5) et conduisant à une réponse très variable à l'effet mâle. Ces observations ainsi que les résultats de cette étude montrent que, sous une même photopériode, les autres facteurs de l'environnement, comme la température, la pluviométrie, le régime alimentaire, ou les facteurs sociaux $(2,16,20)$, jouent un rôle bien plus déterminant dans l'entraînement des rythmes reproductifs chez les chèvres vivant dans les régions subtropicales. Il est également possible que, dans ce cas d'étude, il existe un effet direct de la saison des pluies sur l'apparition du comportement d'œstrus de ces chèvres. Il apparaît dans la répartition de la pluviométrie au cours de l'année d'étude (tableau II) que l'automne, et précisément le mois de novembre, était particulièrement pluvieux comparé à une moyenne de 30 années, comme indiqué au tableau I. Garcia en 1981 (10) a mis en évidence une apparition rapide du comportement d'œstrus quelques jours après le début de la saison humide chez des chèvres vivant sous un environnement aride.

La dissociation entre ovulations et œstrus (ovulations silencieuses ou œstrus non accompagnés d'ovulation) a constitué un élément marquant des cycles sexuels de la chèvre en Tunisie. Ces évènements ont été plus fréquents au début ou à la fin de la saison d'activité sexuelle. Ces résultats sont similaires à ceux trouvés sur d'autres races caprines $(7,21)$. En début de la saison sexuelle, les comportements d'œstrus observés sans ovulations préalables montrent encore une fois la différence entre la brebis et la chèvre. Chez cette dernière, l'œstradiol secrété par les follicules en croissance durant la phase de transition entre l'anœstrus et la saison sexuelle suffit pour déclencher le comportement d'œstrus sans imprégnation préalable par la progestérone comme c'est le cas pour la brebis $(23,24)$.

Le taux d'ovulation subit également une variation tout au long des mois d'activité ovulatoire. Il est maximum au début de la saison sexuelle et il prend des valeurs minimales pendant le dernier tiers de la saison. Les présents résultats rejoignent ceux de Restall (19) qui a également montré que pour les chèvres australiennes, le taux d'ovulation est maximum au début de la saison de reprise de l'activité ovulatoire. En revanche, Derquaoui et El Khaledi ont montré que le taux moyen d'ovulation ne variait pas en fonction du mois chez la chèvre D'Man du Maroc (9). Les valeurs moyennes élevées du taux d'ovulation obtenues en septembre et en octobre confirment le caractère relativement prolifique des chèvres locales (22), bien que la présence continue des mâles dans le présent protocole aurait pu contribuer à augmenter le taux d'ovulation (19). Il est d'ailleurs probable que la présence continue des boucs parmi les chèvres ait pu aussi contribuer à prolonger la saison d'activité ovulatoire (21). Globalement, les présents résultats sur la diminution progressive du taux d'ovulation du début vers la fin de la saison sexuelle expliquent en partie les différences de prolificité observées dans les élevages caprins et permettent d'affirmer que, dans les troupeaux où l'objectif de prolificité est recherché, la mise à la reproduction des chèvres devrait se faire en début de la saison sexuelle.

\section{BIBLIOGRAPHIE}

1. AMMAR-KHODJA F., BRUDIEUX R., 1982. Seasonal variations in the cyclic luteal ovarian activity in the Tadmit ewe in Algeria. J. Reprod. Fert., 65: 305-311.

2. BARIL G., CHEMINEAU P., COGNIE Y., GUERIN Y., LEBOEUF B., ORGEUR P., VALLET J.C., 1993. Manuel de formation pour l'insémination artificielle chez les ovins et les caprins. Rome, Italie, FAO, 231 p. (Prod. Santé Anim. $n^{\circ}$ 83)

3. BODIN L., ELSEN J.M., HANOCQ E., FRANÇOIS D., LAJOUS D., MANFREDI E., MIALON M.M., BOICHARD D., FOULLEY J.L., SANCRISTOBAL-GAUDY M., TEYSSIER J., THIMONIER J., CHEMINEAU P., 1999. Génétique de la reproduction chez les ruminants. Prod. Anim., $12: 87-100$

4. CHARALLAH S., KHAMMAR F., AMIRAT Z., LAKHDARI Y., 2000. Evaluation de I'activité sexuelle mâle et femelle: caractérisation zootechnique et nutritionnelle chez la chèvre Bédouine. In: Actes conférence internationale sur les caprins, Tours, France, 15-21 mai 1990, tome I, p. 460

5. CHEMINEAU P., 1983. Effect on oestrus and ovulation of exposing Creole goats to the male three times of the year. I. Reprod. Fert., 67: 65-72. 6. CHEMINEAU P., 1986. Seasonal behaviour and gonadal activity during the year. I. Female oestrous behaviour and ovarian activity. Reprod. Nutr. Dev., 26: 441-452.

7. CHEMINEAU P., DAVEAU A., MAURICE F., DELGADILLO J.A., 1992. Seasonality of oestrus and ovulation is not modified by subjecting female Alpine goats to a tropical photoperiod. Small Rumin. Res., 8: 299-312.

8. CHEMINEAU P., MALPAUX B., THIERY J.C., VIGNE C., MORELLO H., ZARAZAGA L., PELLETIER J., 1995. The control of seasonality: A challenge to small ruminant breeding. In: Enne G., Greppi F., Lauria A., Eds, Proc. XXX International Symposium of "Società Italiana per il progresso della Zootecnica", Milan, Italy, 11-13 Sept. 1995. Paris, France, Elsevier-Biofutur, p. 225-250. 
9. DERQUAOUI L., EL KHALEDI O., 1994. Evaluation de l'activité sexuelle pendant la saison de baisse de fertilité chez la chèvre de race D'Man. In : $2^{\mathrm{e}}$ conférence African Small Ruminant Research Network, Arusha, Tanzania, 7-11 déc. 1992. Addis-Abeba, Ethiopie, Cipea, p. 49-51.

10. GARCIA B.J.G., 1981. Genetic analysis of a crossbreedding experiment using improved dairy goat breeds and native goats in a dry tropical environment. PhD Thesis, University of California, Davis, USA.

11. HANOCQ E., BODIN L., THIMONIER J., TEYSSIER J., MALPAUX B., CHEMINEAU P., 1999. Genetic parameters of spontaneous spring ovulatory activity in Mérinos d'Arles sheep. Genet. Sel. Evol., 31: 77-90. 12. KARSCH F.J., BITTMAN E.L., FOSTER D.L., GOODMAN R.L., LEGAN S.J., ROBINSON J.E., 1984. Neuroendocrine basis of seasonal reproduction. Recent Prog. Horm. Res., 40: 185-225.

13. KHALDI G., 1984. Variations saisonnières de I'activité ovarienne, du comportement d'œstrus et de la durée de l'anœestrus post-partum des femelles ovines de race Barbarine: Influence du niveau alimentaire et de la présence du mâle. Doct. Etat Sci., université des Sciences et Techniques du Languedoc, Montpellier, France, 168 p.

14. LAHLOU-KASSI A., MARIE M., 1984. Sexual and ovarian function of the D'Man ewe. In: Land R.B., Robinson D.W., Eds, Genetics of reproduction in sheep. London, UK, Butterworths, p. 245-260.

15. LASSOUED N., KHALDI G., 1995. Variations saisonnières de I'activité sexuelle des brebis de races Queue Fine de l'Ouest et Noire de Thibar. Options Méditerr., 6 : 27-34.

16. MANI A.U., MCKELVEY W.A.C., WATSON E.D., 1996. Effect of undernutrition on gonadotrophin profiles in non-pregnant, cycling goats. Anim. Reprod. Sci., 43: 25-33.

\section{Summary}

Lassoued N., Rekik M. Seasonal Variations of Estrus and Ovulation in Native Maure Goats of Tunisia

In an attempt to characterize seasonal variations of ovulation and estrous behavior, 22 adult non-pregnant Maure Tunisian goats were used. The experimental period lasted 14 months during which the goats were run on natural rangeland and supplemented with constant levels of concentrate (200 g/nanny goat/day). The goats were also exposed to natural photoperiod and kept in the presence of males. Aproned bucks were used twice daily to detect estrus, while ovarian activity was monitored monthly by laparoscopy. Throughout the breeding season, which occurred from September to March, $80 \%$ of the nanny goats exhibited estrus at least once and $53 \%$ of all estrous cycles were accompanied by ovulations. This season preceded a period of sexual inactivity (March-August). The incidence of abnormal short estrous cycles was higher at the end of the breeding season. During the month of September, $86 \%$ of the goats displayed estrus. A similar seasonal distribution was observed for cyclic ovarian activity, with the proportion of ovulating goats increasing gradually from September to reach $100 \%$ during December, and then declining to reach a minimum of $14 \%$ during March. The mean ovulation rate was $1.51 \pm 0.29$ with a maximum of $1.94 \pm 0.74$ at the start of the breeding season in September, and a minimum of $1.25 \pm 0.50$ at the end of this season in March. There was a zero evolution rate between April and August included and there was a significant difference $(P<0.05)$ between the start of the breeding season and the end. It was concluded that native goats in Tunisia, under semiarid grazing conditions, exhibited a seasonal pattern of reproduction.

Keywords: Nannygoat - Oestrus cycle - Ovulation - Breeding season - Reproduction - Tunisia.
17. MAULEON P., DAUZIER L., 1965. Variations de la durée de I'anœstrus de lactation chez les brebis de race lle de France. Ann. Biol. Anim. Bioch. Biophys., $5:$ 131-143.

18. OLDHAM C.M., LINDSAY D.R., 1980. Laparoscopy in the ewe: a photographic record of the ovarian activity of ewes experiencing normal or abnormal oestrous cycles. Anim. Reprod. Sci., 3: 119-124.

19. RESTALL B.J., 1992. Seasonal variation in reproductive activity in Australian goats. Anim. Reprod. Sci., 27: 305-318.

20. RESTALL B.J., RESTALL H., WALKDEN-BROWN S.W., 1995. The induction of ovulation in anovulatory goats by oestrous females. Anim. Reprod. Sci., 40: 299-303.

21. RIVERA G.M., ALANIS G.A., CHAVES M.A., FERRERO S.B., MORELLO H.H., 2003. Seasonality of estrus and ovulation in Creole goats of Argentina. Small Rumin. Res., 48: 109-117.

22. STEIBACH J., 1988. Experiences with the evaluation of the production potential of local and imported goat breeds in northern Tunisia. Anim. Res. Dev., 28: 100-114.

23. SUTHERLAND S.R.D., 1987. Progesterone and estrogen requirements for oestrous behaviour in goat and sheep. Proc. $4^{\text {th }}$ AAAP Anim. Sci. Congress, Hamilton, New Zealand, 1-6 Feb., p. 227.

24. SUTHERLAND S.R.D., 1988. Seasonal breeding and oestrus in the female goat. PhD Thesis, University of Western Australia, Perth, p. 116.

25. THIMONIER J., MAULEON P., 1969. Variations saisonnières du comportement d'œstrus et des activités ovarienne et hypophysaire chez les ovins. Ann. Biol. Anim. Bioch. Biophys., 9: 233-250.

Reçu le 26.02.2004, accepté le 05.07.2005

\section{Resumen}

Lassoued N., Rekik M. Variaciones estacionarias del estro y de la ovulación en la cabra local Maura en Tunisia

Un total de 22 cabras Mauras tunisinas permitieron caracterizar las variaciones estacionarias del estro y de la ovulación en un periodo de 14 meses. Las cabras se mantuvieron no grávidas durante el periodo experimental. Fueron conducidas por senderos naturales y recibieron un aporte constante en concentrado de 200 g por cabra por día. Fueron también expuestas a un fotoperiodo natural y mantenidas en presencia constante de machos. Se utilizaron machos marcadores para la detección bi cotidiana del comportamiento del estro, mientras que el examen de la actividad ovárica fue realizado por endoscopías mensuales. En el curso de la estación reproductiva, que se extendió de septiembre a marzo, $80 \%$ de las cabras mostraron un comportamiento de estro al menos una vez y $53 \%$ de la totalidad de los ciclos estrales fueron acompañados de ovulación. Esta estación fue precedida por un periodo de inactividad sexual de marzo a agosto. La incidencia de los ciclos estrales anormales, de corta duración fue más elevada hacia el fin de la estación reproductiva. Durante el mes de setiembre, $86 \%$ de las cabras mostraron un comportamiento de estro. La misma distribución estacionaria fue también observada en la actividad cíclica del ovario. La proporción de hembras ovulando durante la estación reproductiva aumentó progresivamente a partir del mes de setiembre, alcanzando un máximo de $100 \%$ en diciembre, seguido de una disminución con un mínimo de $14 \%$ en el mes de marzo. El valor promedio de la tasa de ovulacion fue de 1,51 $\pm 0,29$, alcanzando un máximo de 1,94 $\pm 0,74$ en el inicio de la estación reproductiva en setiembre y un mínimo de 1,25 \pm 0,50 al fin de esta estación, durante el mes de marzo. La tasa de ovulación fue nula entre los meses de avril y agosto incluídos. La distancia entre el inicio y el fin de la estación reproductiva fue significativamente diferente $(p<0,05)$. En conclusión, las cabras locales en Tunisia, en un medio semi árido, tienen un ritmo reproductivo estacionario.

Palabras clave: Caprino - Cabra - Ciclo estral - Ovulación Epoca de apareamiento - Reproducción - Túnez. 